\title{
Entwicklung spezifischer Kinase-Hemmer für die Therapie entzündlicher Erkrankungen
}

\author{
G. Neubauer $^{1}$ \\ ${ }^{1}$ Cellzome, a GlaxoSmithKline (GSK) company, Heidelberg
}

Kinasen als Enzymklasse in der Wirkstoffforschung Kinasen sind eine wichtige Enzymklasse, die in Signalwegen normale Zellfunktionen regeln und in vielen Erkrankungen (z.B. bei unterschiedlichen Krebsarten, Entzündungskrankheiten oder Herz-Kreislauf-Erkrankungen) dysreguliert sind. Die Wichtigkeit der Kinasen als Drug Targets (Zielproteine für Wirkstoffe) zeigt sich daran, dass 20-30\% der Forschungs- und Entwicklungsprojekte in der pharmazeutischen Industrie Kinase-Projekte sind. Fast 10 Jahre nach der Zulassung des ersten niedermolekularen Kinase-Inhibitors zur zielgerichteten Therapie in der Onkologie befindet sich nun der erste Kinase-Hemmer für Entzündungskrankheiten im Zulassungsverfahren. Dies steht im Kontrast zu den mehr als 15 Kinase-Inhibitoren für onkologische Indikationen, die seither auf den Markt gebracht wurden [1]. Die Relevanz von Kinasen als zentralen Schaltstellen in der Signaltransduktion des Immunsystems ist seit Langem unbestritten, der Mangel an zugelassenen Inhibitoren für inflammatorische Erkrankungen reflektiert jedoch die Schwierigkeit, adäquate, selektive Arzneistoffe zu entwickeln. Während bei Therapien in der Onkologie ein hohes Risiko an Nebenwirkungen tolerierbar ist, muss dieses Risiko bei chronischen Indikationen auf ein absolutes Minimum reduziert werden, weshalb eine hohe Spezifität des Inhibitors für die Ziel-Kinase unabdingbar ist. Dies ist bei Kinasen eine hohe Hürde, da die Enzymfamilie mit 518 Mitgliedern sehr groß ist und sie eine hohe Homologie in der ATP-Bindungstasche, der Bindungsstelle der meisten Inhibitoren, aufweisen. Mit Tofacitinib (Pfizer) befindet sich nun ein Kinase-Inhibitor in der Zulassungsphase, der von den 518 Kinasen nur die 4 Januskinasen (JAK1,2,3 und Tyk2) inhibiert. Die funktionelle Inhibition von hauptsächlich JAK1 und JAK3 führt dazu, dass eine zentrale Schaltstelle im intrazellulären Netzwerk der proinflammatorischen Zytokinausschüttung ausgeschaltet wird, was zu den positiven klinischen Studienergebnissen für rheumatoide Arthritis führte (www.clinicaltrials.com).

PI3Ky als Target für Entzündungskrankheiten PI3Kr (Phosphatidyl-Inositol-3-Kinase gamma) ist eine Lipid/ Protein-Kinase, die ebenfalls eine zentrale Rolle in der Signalweiterleitung in Immunzellen einnimmt. PI3Kr ist eine von insgesamt 4 Klasse-1-PI3-Kinasen, die alle im Visier der pharmazeutischen Wirkstoffforschung stehen. Die 4 eng verwandten Kinasen (PI3K $\alpha, \beta, \delta, \gamma)$ phosphorylieren Phosphatidylinositol $(4,5)$ phosphat $\left(\mathrm{PI}(4,5) \mathrm{P}_{2}\right)$ zu PI $(3,4,5) \mathrm{P}_{3}$, einem sekundären Botenstoff, der wiederum eine wichtige Rolle in vielen Signalwegen der Zellproliferation, -differenzierung und -migration spielt [2]. PI3K $\gamma$ kommt hauptsächlich in Immunzellen vor, und genetische oder pharmakologische Inak- 
tivierung dieser Kinase führt zu unterschiedlichen antiinflammatorischen Effekten, vor allen Dingen des angeborenen Immunsystems. So wird z. B. die Chemotaxis von Immunzellen [3] und die Degranulierung von Mastzellen [4] durch die Inhibition von PI3Kr gehemmt. Während die genetische Inaktivierung in Knockout-Mäusen wichtige Einsichten in die Biologie von PI3Kr lieferte [5], fehlte bisher ein selektiver pharmakologischer Inhibitor, der die Erprobung in humanen Primärzellen ermöglicht und damit die Rolle von PI3Kr im menschlichen Immunsystem klärt.

\section{Chemoproteomik zur Identifizierung selektiver Inhibitoren von PI3Ky}

Um die Selektivität von Kinase-Inhibitoren in physiologisch relevanten Systemen messen zu können, haben wir eine chemoproteomische Plattform entwickelt Kinobeads $^{\mathrm{TM}}$ ) [6], die statt rekombinanter Proteine Zell- oder Gewebeextrakte nutzt. Dadurch liegen die Zielproteine in voller Länge, mit den physiologischen posttranslationalen Modifikationen und Proteinkomplexen vor. Die Aktivität von Inhibitoren wird in einem Kompetitionsbindungs-Assay gemessen, in dem der Inhibitor konzentrationsabhängig mit einer speziell entwickelten, kinasebindenden Matrix (den Kinobeads ${ }^{T M}$ ) um die Bindung der Zielkinasen direkt im Zellextrakt kompetitiert. Die Detektion der Kinasen erfolgt anschließend durch quantitative Massenspektrometrie und erzielt ein umfassendes Selektivitätsprofil des Inhibitors für alle in dieser Zelle vorliegende Kinasen.

Um selektive Wirkstoffkandidaten für PI3K $\gamma$ zu finden, haben wir die Kinobeads ${ }^{\mathrm{TM}}$-Plattform erweitert. Zum einen wurde eine neue Kinobeads ${ }^{T M}$-Matrix geschaffen, die die Familie der PIKinasen bindet, und zum zweiten musste ein Testsystem etabliert werden, das einen höheren Durchsatz an Substanzen bewältigen kann. Dazu haben wir statt der massenspektrometrischen Detektion einen antikörperbasierten Ansatz auf Arrays entwickelt. Mit dieser Methode können nun ganze Bibliotheken mit 100000 Substanzen gegen bis zu 10 unterschiedlichen Zielproteinen in einem Assay getestet werden. Die koordinierte Nutzung beider Systeme führte zu der Identifikation des ersten selektiven PI3K $\gamma$-Inhibitors, der antiinflammatorische Wirkung in Tiermodellen zeigt [7]. Damit konnte bestätigt werden, dass die Hemmung der Kinase-Aktivität von PI3Kr eine valide Strategie zur therapeutischen Intervention bei Entzündungskrankheiten darstellt. Obwohl die Rolle von PI3Kr im angeborenen Immunsystem relativ gut beschrieben ist, konnte bisher noch nicht eindeutig gezeigt werden, dass PI3Kr auch eine Rolle im adaptiven Immunsystem innehat. In einem zellbasierten Aktivitätsprofil von humanen Primärzellen unter proinflammatorischen Stimuli konnte die Selektivität von CZC24832 bestätigt und außerdem eine hemmende Wirkung der Ausschüttung von Interleukin 17A (IL-17A) gezeigt werden. Weiterführende Studien konnten diesen Effekt auf die Differenzierung von naiven T-Zellen zu $T_{H} 17$-Zellen zurückführen, die durch $\mathrm{PI} 3 \mathrm{~K} \gamma$ reguliert wird. Die antiinflammatorische Wirkung von PI3Kr-Inhibitoren kann also durch die Modulierung von angeborenen und adaptiven Immunreaktionen herrühren. Das erstmalige Aufzeigen der Regulierung der Differenzierung von $\mathrm{T}_{\mathrm{H}} 17$-Zellen durch PI3Kr wird für Krankheitsbilder interessant sein, die stark durch IL-17 geprägt sind.

Interessenkonflikte: Dr. Gitte Neubauer ist Geschäftsführerin der Cellzome $\mathrm{GmbH}$, deren einziger Gesellschafter GlaxoSmithKline ist.

\section{Literatur}

1 Fabbro $D$ et al. Targeting cancer with small-molecular-weight kinase inhibitors. Methods Mol Biol 2012; 795: 1-34

2 Vanhaesebroeck $B$ et al. The emerging mechanisms of isoform-specific PI3K signalling. Nat Rev Mol Cell Biol 2010; 11: 329-341

3 Hawkins PT et al. Signalling through Class I PI3Ks in mammalian cells. Biochem Soc Trans 2006; 34: 647-662

4 Bohnacker $T$ et al. PI3Kgamma adaptor subunits define coupling to degranulation and cell motility by distinct $\operatorname{PtdIns}(3,4,5) \mathrm{P} 3$ pools in mast cells. Sci Signal 2009; 2: ra27

5 Hirsch $E$ et al. Central role for G protein-coupled phosphoinositide 3kinase gamma in inflammation. Science 2000; 287: 1049-1053

6 Bantscheff $M$ et al. Quantitative chemical proteomics reveals mechanisms of action of clinical ABL kinase inhibitors. Nat Biotechnol 2007; 25: $1035-1044$

7 Bergamini $G$ et al. A selective inhibitor reveals PI3Kgamma dependence of T(H)17 cell differentiation. Nat Chem Biol 2012; 8: 737

Bibliografie

DOI http://dx.doi.org/10.1055/s-0032-1324912

Arzneimittelforschung 2012; 62, Suppl. 1: S18-S19

(c) Georg Thieme Verlag KG Stuttgart · New York .

ISSN 0004-4172 